\title{
Defining the characteristics of
} intermediate care models including transitional care: an international Delphi study

Sezgin, D

http://hdl.handle.net/10026.1/16330

10.1007/s40520-020-01579-z

Aging Clinical and Experimental Research

Springer Science and Business Media LLC

All content in PEARL is protected by copyright law. Author manuscripts are made available in accordance with publisher policies. Please cite only the published version using the details provided on the item record or document. In the absence of an open licence (e.g. Creative Commons), permissions for further reuse of content should be sought from the publisher or author. 


\title{
Defining the characteristics of intermediate care models including transitional care: an international Delphi study
}

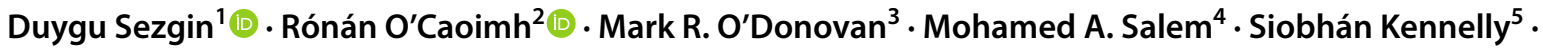 \\ Luz Lopez Samaniego ${ }^{6}$. Cristina Arnal Carda ${ }^{7} \cdot$ Rafael Rodriguez-Acuña $^{6} \cdot$ Marco Inzitari $^{8} \cdot$ Teija Hammar $^{9}$. \\ Claire Holditch $^{10}$. Janet Prvu Bettger ${ }^{11}$. Martin Vernon ${ }^{12,13}$. Áine Carroll ${ }^{14,15}$. Felix Gradinger ${ }^{16,17}$. \\ Gaston Perman $^{18}$. Martin Wilson ${ }^{19}$. Antoine Vella ${ }^{20}$. Antonio Cherubini ${ }^{21}$. Helen Tucker $^{22}$. Maria Pia Fantini ${ }^{23}$. \\ Graziano Onder ${ }^{24}$. Regina Roller-Wirnsberger ${ }^{25}$. Luis Miguel Gutiérrez-Robledo ${ }^{26}$. Matteo Cesari ${ }^{27}$. \\ Paula Bertoluci ${ }^{28} \cdot$ Magdalena Kieliszek $^{29}$. Wilma van der Vlegel-Brouwer ${ }^{30} \cdot$ Michelle Nelson $^{31,32}$. \\ Leocadio Rodríguez Mañas ${ }^{33}$. Eleftheria Antoniadou ${ }^{34}$. François Barriere ${ }^{35}$. Sebastian Lindblom ${ }^{36}$. Grace Park ${ }^{37}$. \\ Isidoro Pérez ${ }^{38}$. Dolores Alguacil ${ }^{39}$. Douglas Lowdon ${ }^{40}$. Maria Eugenia Alkiza ${ }^{41}$. Cristina Alonso Bouzon ${ }^{42}$. \\ John Young ${ }^{43}$. Ana Carriazo ${ }^{44}$. Aaron Liew ${ }^{45,46}$. Anne Hendry ${ }^{47,48}$ on behalf of European Union Advantage Joint \\ Action Work Package 7 partners in collaboration with the International Foundation for Integrated Care Special \\ Interest Group on Intermediate Care
}

Received: 16 April 2020 / Accepted: 25 April 2020

(c) Springer Nature Switzerland AG 2020

\begin{abstract}
Background Although there is growing utilisation of intermediate care to improve the health and well-being of older adults with complex care needs, there is no international agreement on how it is defined, limiting comparability between studies and reducing the ability to scale effective interventions.

Aim To identify and define the characteristics of intermediate care models.

Methods A scoping review, a modified two-round electronic Delphi study involving 27 multi-professional experts from 13 countries, and a virtual consensus meeting were conducted.

Results Sixty-six records were included in the scoping review, which identified four main themes: transitions, components, benefits and interchangeability. These formed the basis of the first round of the Delphi survey. After Round 2, 16 statements were agreed, refined and collapsed further. Consensus was established for 10 statements addressing the definitions, purpose, target populations, approach to care and organisation of intermediate care models.

Discussion There was agreement that intermediate care represents time-limited services which ensure continuity and quality of care, promote recovery, restore independence and confidence at the interface between home and acute services, with transitional care representing a subset of intermediate care. Models are best delivered by an interdisciplinary team within an integrated health and social care system where a single contact point optimises service access, communication and coordination. Conclusions This study identified key defining features of intermediate care to improve understanding and to support comparisons between models and studies evaluating them. More research is required to develop operational definitions for use in different healthcare systems.
\end{abstract}

Keywords Delphi technique $\cdot$ Definition $\cdot$ Older adults $\cdot$ Intermediate care $\cdot$ Transitional care $\cdot$ Integrated care $\cdot$ Care models

Rónán O’Caoimh, Aaron Liew and Anne Hendry are co-senior authors.

Electronic supplementary material The online version of this article (https://doi.org/10.1007/s40520-020-01579-z) contains supplementary material, which is available to authorized users.

Extended author information available on the last page of the article

\section{Introduction}

The coordination and integration of care for older people is increasingly recognised as important to reduce miscommunication, duplication, omission errors, patient dissatisfaction and reduce unnecessary healthcare utilisation [1]. Inefficient 
processes to ensure smooth transfers of care are common, costly and potentially harmful $[2,3]$, often resulting in recurrent hospital attendances and (re)admissions [4]. Older and frail patients appear to be particularly at risk of adverse events related to discharge and transitions of care [5]. This relates in part to the frequency with which they experience care transitions but also because systems are not attuned to meet their needs [6]. Healthcare systems that provide vertically integrated models, foster interdisciplinary relationships and encourage the involvement of older people and their families are considered the optimal approach to minimise adverse outcomes [6,7].

Intermediate care services are designed to provide continuity of care and are often used to enhance recovery, promote ability, optimise management and prevent adverse outcomes including functional decline among older people, particularly those with chronic disease and frailty [8-10]. Elements of these models of care include comprehensive assessment, enablement and rehabilitation, which target the restoration of health and the optimisation of function, activity and participation [8, 9, 11, 12]. These can play an important role in times of change in health states as older people transition between services such as moving between home (community residence) and hospital, assisted living or institutional care [13, 14]. Intermediate care including transitional care can contribute to the continuum of services that enable older adults to recover or maintain independence following a period of illness, injury or hospitalisation $[11,15]$. Although results for its effectiveness are as yet mixed $[8,16]$, there is growing evidence that this approach to providing care can improve function [17-20], lower costs [21-28] and reduce healthcare utilisation [27-31] for older adults.

Despite its increasing use, there remains ambiguity over how intermediate care is defined [32] in both the literature and in clinical practice. This may be due to differences in the organisation and operation of healthcare services in different geographical areas [9]. The lack of clarity over definitions makes the interpretation of research findings and comparison of models in clinical practice challenging [4]. To aid healthcare practitioners and policy-makers, there is a need to establish globally accepted terminology for this approach to care [32, 33]. Given these points, we aimed to identify key defining features of different intermediate care models using an international Delphi consensus approach.

\section{Methods}

This study was conducted as part of the ADVANTAGE Joint Action on Frailty Prevention by the members of Work Package 7 [16]. The study consisted of two phases, an initial scoping review, conducted to identify existing descriptions of intermediate care, followed by a two-round electronic Delphi survey with a virtual consensus meeting. The methods flow chart is presented in Fig. 1.
Fig. 1 Methods flow chart

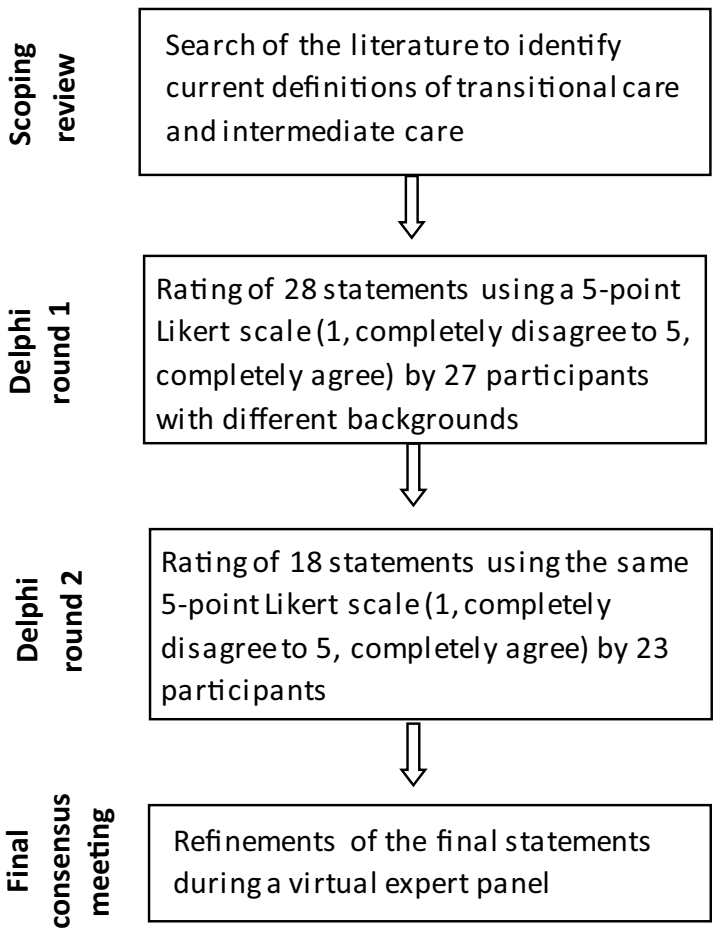

66 articles were identified. Qualitative data synthesis resulted in the generation of themes. Four main themes were identified: transitions, components, benefits and interchangeability
16 statements met the threshold for inclusion *(See figure 2). These were forwarded to the final consensus meeting 


\section{Phase 1: scoping review}

Scoping reviews help in synthesising data related to research questions by refining key concepts of existing evidence [34]. We sought to identify available definitions of intermediate care prior to conducting the Delphi study using a methodological framework for conducting scoping reviews recommended by Levac et al. [35]. First, the problem was identified, and the following research question was investigated: "What are the current definitions of intermediate care in the literature?" Preferred Reporting Items for Systematic reviews and Meta-Analyses extension for Scoping Reviews (PRISMA-ScR) checklist was used for reporting processes [36].

\section{Data sources and selection process}

CINAHL, Cochrane Library, EMBASE and PubMed databases were searched. The following search string was used: (((("Intermediate care" OR "transitional care")) AND ("hospital at home" OR "home care" OR "residential care" OR "reablement" OR "independ*" OR "readmission" OR "Prolonged stay" OR "long term" OR "community care"))). An additional search on OpenGrey included the search terms, intermediate care and transitional care. Reference tracking of systematic reviews or other review papers was also performed. Papers were included if they were (1) research studies, review articles or grey literature that reported a definition of transitional and/or intermediate care as primary or secondary sources, and (2) published between 1 January 2002 and 5 February 2019. The search was initially planned to be limited to 2002-2017 (the previous 15 years, consistent with the state-of-theart reviews for the Joint Action on Frailty Prevention (ADVANTAGE)) but the search was extended to 2019 for this study. Conference proceedings, study protocols and any studies or reports that did not meet these criteria were excluded.

\section{Data extraction and analysis}

Titles and abstracts of the identified records were independently screened by a core team of reviewers according to the inclusion and exclusion criteria. Data were extracted by the same reviewers using a standardised template as direct quotations as reported in the articles (i.e. first-order constructs), pooled, refined and synthesised accordingly. A qualitative (thematic) analysis approach [37] using NVivo was performed to analyse the data; extracted data were coded, from which main and sub-themes emerged relating to the definition, concepts and components of intermediate care.

\section{Phase 2: Delphi study and consensus meeting}

A Delphi study design systematically combines the views of experts to reach consensus on a particular topic [38]. To reach agreement on the nature of intermediate care, a broad range of experts including academics, practitioners and policy-makers were included. A modified Delphi study consisting of a two-round electronic survey (e-Delphi process) followed by a virtual consensus meeting was conducted $[39,40]$. The e-Delphi approach was chosen to bring experts together from different countries via online surveys and meetings.

\section{Participants}

A non-probabilistic sampling approach was followed. In total, 55 experts from 17 countries were sent invitations to participate in the Delphi. Invitees either had a health and social care policy role, had published research or audit on the topic, or were practitioners who had expertise in intermediate or transitional care services or frailty. Seven of these were drawn from the Joint Action on Frailty Prevention's (ADVANTAGE), Work Package 7, examining models of integrated care for managing and preventing frailty [41]. Additional external experts were included in the consensus meeting to refine and group statements. For this purpose, members of the International Foundation for Integrated Care (IFIC) Special Interest Group (SIG) on Intermediate Care were invited to participate in the virtual meeting. The IFIC SIG on intermediate care is composed of experts from various disciplines and countries to collaborate, exchange insights and enhance their knowledge in the area of intermediate care.

\section{Ethics}

All participants agreed to participate and provided informed consent electronically before proceeding with the eDelphi surveys. Participants' responses were protected behind a firewall accessible only to the core research team. Confidentiality was maintained according to the European Union General Data Protection (2018) regulations.

\section{Data collection}

The Delphi surveys were open between the 1-15 July 2019 (first round) and 22 July-12 August 2019 (second round). Weekly reminders were sent to the participants. Using a fiveitem Likert scale (1, completely disagree to 5 , completely agree), participants were asked to rate the statements generated in the scoping review. They were also given the opportunity to add comments. In Round 2, participants rated the revised statements. 
The virtual consensus meeting was conducted as two sessions (morning and afternoon) due to time zone differences between the participant countries. Meetings were moderated by a member of the core research group. Outcomes of the first session were shared with the participants of the second meeting prior to commencing the discussion. All statements and their agreement levels (expressed as percentage) were read out by another member of the core team, which started the group discussions. Discussions focused on the rewording and merging of statements rather than altering the consensus reached by the eDelphi panel. All refinements were made by majority agreement. The emphasis was, therefore, on refining accepted statements to minimise repetition or duplication.

\section{Data analysis}

Survey data were analysed using simple descriptive statistics. In both rounds, statements scored as 4 (agree) or 5 (completely agree) by $70 \%$ or more of participants or where less than $15 \%$ of participants scored the statement as 1 (completely disagree) or 2 (disagree) [42] were included. A cutoff of $70 \%$ was selected based on group consensus similar to the most widely used cut point in Delphi studies, $75 \%$ [43]. Statements with ratings of 3 or below were excluded. In Round 1, qualitative methods were used for the analysis of comments added by the participants. For this purpose, all comments were pooled, grouped under common themes and then mapped under relevant statements. Revised statements were then re-circulated in the following round for re-rating. All qualitative comments were mapped under relevant statements considering whether they supported them or not. Comments that were not directly related to the statements but addressed definitions of intermediate care were classified to generate new statements. The data analysis strategy is summarised in Fig. 2.

\section{Results}

\section{Scoping review}

The literature search yielded 1891 records in total. Cross-referencing of systematic reviews and narrative review articles resulted in 79 additional citations. A search of OpenGrey added a further 14 reports, resulting in a total of 1984 articles for screening. Further details of the screening process are outlined in a PRISMA flow diagram in supplementary Fig. 1. Records that did not meet the inclusion criteria or were identified as duplicates were excluded $(n=1762)$. Therefore, 222 full-text articles were assessed for eligibility. Following this, a further 156 records were excluded as they did not report a definition $(n=94)$, were conference abstracts $(n=30)$, were not related to intermediate care $(n=29)$, or were study protocols $(n=3)$. In total, 66 articles provided a definition of intermediate care models, including subtypes such as transitional care, and, therefore, were included. These definitions included both primary (original) definitions and citations from secondary sources. Analysis of the definitions included in the scoping review identified four main themes: transitions, components, benefits and interchangeability (Fig. 3). The first theme "transitions" referred to care transitions from one setting or level of care to another. The second theme "components" addressed the characteristics of intermediate care including that is multidisciplinary and comprehensive but time limited, requires coordination, includes a wide range of services, targets highrisk populations, has flexibility for adapting delivery, and

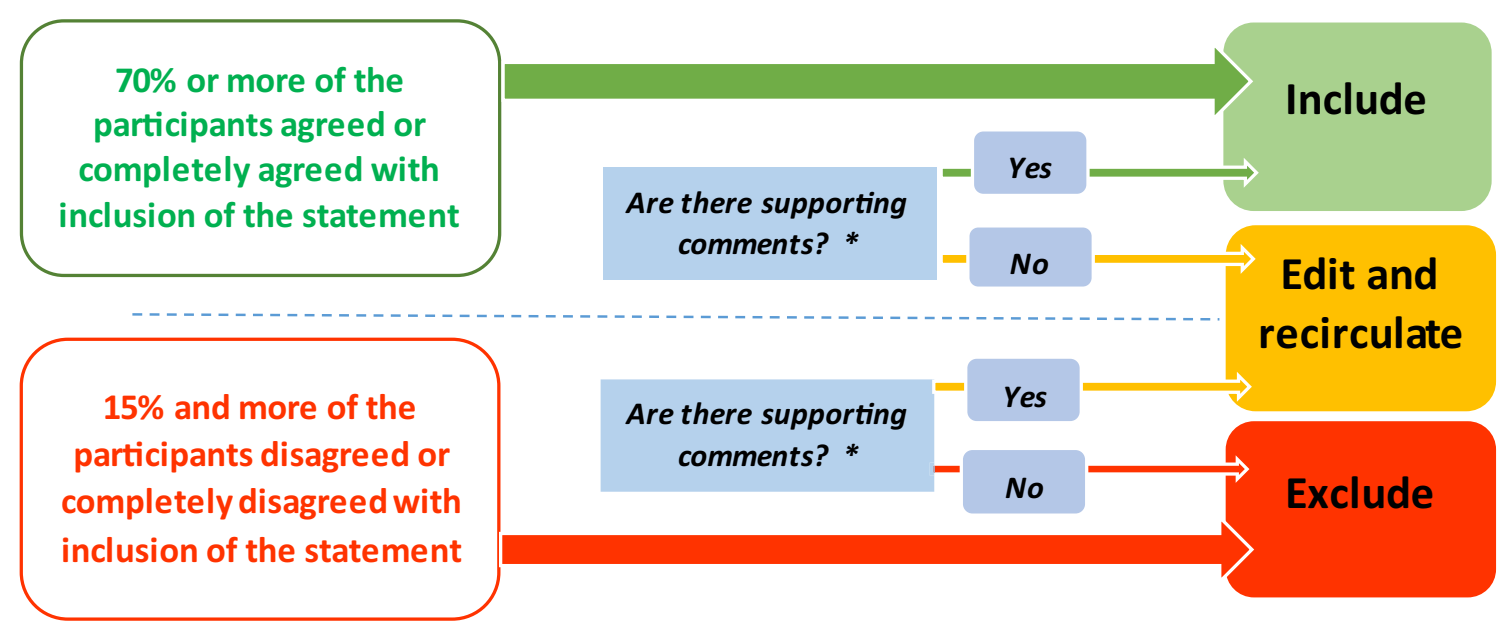

Fig. 2 Analysis strategy for round one and two of the e-Delphi (*Did not apply to the Round 2) 


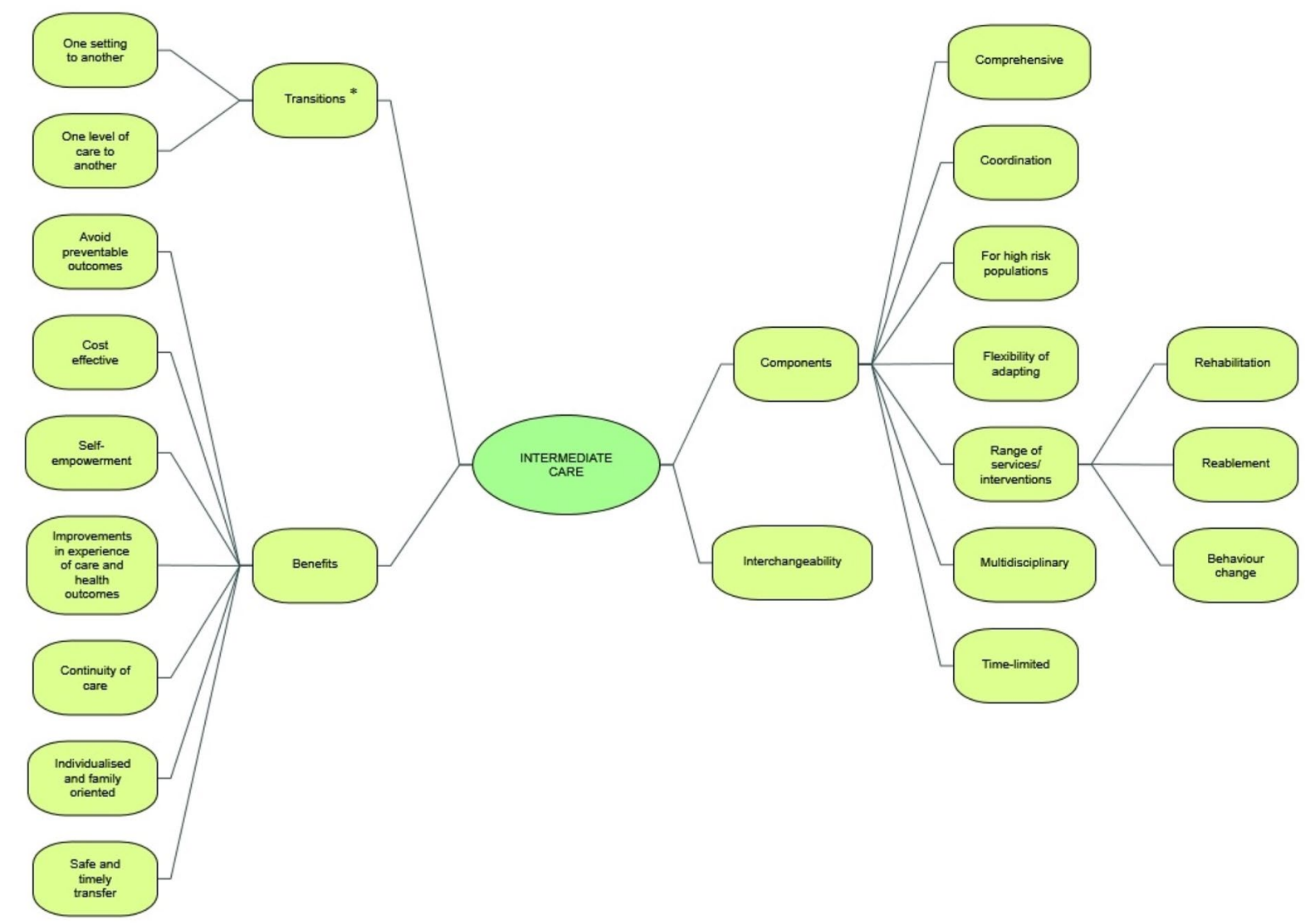

*Includes transitional care as a subset of intermediate care

Fig. 3 Concept map of the definitions of intermediate care pooled from the literature. *Includes transitional care as a subset of intermediate care

considers person-centred care. The third theme "benefits" denoted the positive outcomes of intermediate care including the continuity of care, cost-effectiveness, self-empowerment, individualised and family-oriented care. The fourth theme "interchangeability" referred to the observed use of intermediate care including transitional care, and other similar terms, and the lack of a clear definition of intermediate care in the literature. Following discussion and refinement by the core members, 28 high-level statements were forwarded to the Delphi process.

\section{Delphi Round 1}

A total of 27 experts from 13 countries (Argentina, Austria, Canada, England, Finland, Greece, Ireland, Italy, Malta, Mexico, Scotland, Spain, and the USA), participated in Round 1, giving a response rate of $49 \%$. Round 1 statements were related to the concept and core components of intermediate care, their purpose and benefits, interchangeability of the terms used, and their role in transitions of care. Twentyone of these statements met the threshold and were retained. Most (four out of seven) that did not reach the threshold for inclusion were from the "components/concept" category. A total of 86 comments were received, which were used for editing or merging current statements, or deriving new statements. This reduced the number of included statements to 11 and resulted in the generation of seven new statements for Round 2 (Table 1). The 18 statements were re-categorised into five sections: purpose and concept $(n=2)$; common features between intermediate and transitional care $(n=2)$; differences between intermediate care and transitional care $(n=2)$; types of intermediate care $(n=2)$; and effective organisation of intermediate care $(n=10)$.

\section{Delphi Round 2}

All who took part in Round 1 were invited back for Round 2. Of these, 23 (85\%) participated in Round 2. These were from 11 countries (Argentina, Austria, Canada, England, Finland, Greece, Ireland, Italy, Malta, Mexico, and Spain). In Round 2, the participants disagreed with two of the remaining 18 statements: "Some types of intermediate care, i.e. homebased rehabilitation and reablement can be cost-efficient" and "The main aim of transitional care is to improve the 
Table 1 Final consensus statement on the definitions, target population, approach to care and the organisational structure of intermediate care

How are these models of care defined?

1. Intermediate care is are a broad range of time-limited services, from crisis response to support for several weeks or months, that aim to ensure continuity and quality of care and promote recovery at the interface between hospital and home, care home, primary care and community services

2. Intermediate care, at home or in intermediate care beds, aims to enable recovery, restore independence and confidence, or prevent a decline in functional ability at times of change in health

3. Transitional care services are a subset of intermediate care designed to enable safe, coordinated and timely transfers between care settings Who may benefit?

4. These services may particularly benefit persons who have complex needs or circumstances, are vulnerable to a decline in health status or functional ability, or are at increased risk of (re)admissions to hospital or institutionalisation

What is the approach to care?

5. Intermediate care is based on principles of holistic comprehensive assessment; person-centred care planning; education for patients, family and caregivers; support for self-management (including nutrition and medication management); and continuity and coordination of care. The use of a reablement approach and interdisciplinary rehabilitation has a specific role for persons who have experienced a decline in functional ability

6. The nature, duration and intensity of the multi-dimensional interventions are tailored to the needs of the individual, in collaboration with their family and caregivers, and may involve a case management approach for the duration of the episode

7. Health professionals should adopt relational approaches, creative solutions and simple technologies that enable and support patients, their families and caregivers to be fully involved in care planning, goal setting and monitoring from the earliest stages

How should services be organised?

8. Intermediate care is best delivered by an interdisciplinary team within an integrated health and social care system that links different providers and levels of care in a collaborative network of care and support that includes partners from community and voluntary sectors

9. A single point of contact helps to optimise service access, communication and coordination of care

10. To be effective, intermediate care services should have sufficient capacity and responsiveness, appropriate expertise, clear governance arrangements, and opportunities for education and training to support team members to work collaboratively and to continually improve service quality and outcomes for people and care systems

quality and continuity of care as the individual moves between care settings." (Supplementary Table 1). The results of Round 2 were forwarded to the participants for further discussion in the virtual consensus meeting.

\section{Virtual consensus meeting}

While all Delphi participants were invited to the virtual consensus meeting, only two could attend (one in the morning and one in the afternoon session). They were joined by six external experts from Brazil, France, The Netherlands, Ireland, Poland, and Sweden. The morning meeting was conducted with nine participants (one Delphi participant, four external experts and four core team members). The afternoon session included eight participants (one Delphi participant, two external experts and five core team members). Following the virtual consensus meeting, statements were further refined to avoid duplication or repetition and reduced from 16 to 10 statements distributed under four headings: "How are these models of care defined?"; "Who may benefit?"; "What is the approach to care?"; and "How should services be organised?". Delphi participants and the external experts were asked to comment on these 10 statements. Revisions were applied following their feedback, and then a final consensus statement was approved by all participants (Table 1).

\section{Discussion}

This study applying a scoping literature review combined with a modified e-Delphi design aimed to build consensus towards clear and distinctive definitions of intermediate care services. These services, which are increasingly common, are provided to older adults with multimorbidity and complex healthcare needs who require longer periods for recovery, or specialist care and rehabilitation at home or closer to home $[9,11]$. This paper is, to our knowledge, the first to examine the terminology around intermediate care using a Delphi method. It offers guidance to policymakers, researchers and practitioners by providing insights from international experts into many of the defining features of these services. The study produced agreement and generated consensus statements addressing the definition of intermediate care, and also described important features, which characterise these models including areas of overlap in their target population, approach to care, and organisation. Each of these aspects are outlined and discussed separately below. 


\section{Definitions and purpose}

It was agreed that intermediate care refers to a broad range of time-limited services that aim to provide continuity, improve the quality of care, promote recovery, restore independence and confidence, and prevent decline in functional ability. Intermediate care was described as operating at the interface between home and hospital or institutional care services, consistent with the Steiner et al. [44] definition of intermediate care services as "a range of services that facilitate the transition from hospital to home...". The American Geriatrics Society further states that the settings where these models are best used includes, but is not limited to, hospitals, sub-acute and post-acute nursing facilities, the patient's home, primary and specialty care offices, and long-term care facilities [14]. All participants agreed that intermediate care services are structured and multi-component interventions that are more than a means to facilitate discharges, reflecting a recent Cochrane review by Griffiths et al. [45] that described intermediate care interventions as an intrinsic component of care extending beyond discharge planning.

The discussions noted that the purpose of these models often depends on the specific needs of individual patients, which may change over time. Indeed, the literature suggests that successful transitional care programmes should consider the changing needs of individuals at both pre- and post-discharge stages [14, 46]. A recent concept analysis found that individual and family characteristics such as health status, socioeconomic factors, expectations and habits, inpatient care system, and social factors including the ability of caregivers and support mechanisms, play a major role in the design and application of transitional care [47]. Participants agreed that intermediate care differs from chronic case management since it is time limited. All participants agreed that common features include an aim to improve continuity, flow and safe and timely coordination of care. Consensus suggested that intermediate care aims to enable recovery, restore independence and confidence, or prevent a decline in functional ability at times of change in health. Transitional care can be considered as a specific subset of intermediate care designed to enable safe, coordinated and timely transfers between care settings.

The Delphi found that there are specific challenges in standardising the classification of intermediate care services between different countries and healthcare systems [3, 13]. Discussion of different examples of intermediate care services presented during the consensus meeting highlighted that the language used to describe these services may not always transfer between countries.

\section{Target population}

In the consensus meeting, some participants considered it would be unhelpful to specify the type of patients who would benefit from these services as this may evolve over time and needs to be considered in the context of the other community and hospital services available in the local health and care system. However, it was agreed that intermediate care models, and the subsets that are transitional care services, may particularly benefit individuals who have complex care needs or circumstances, are vulnerable to a decline in health status or functional ability, or at increased risk of (re)admissions to hospital or long-term care. This is consistent with the literature [14] and with the view that older adults, particularly those who are frail with complex healthcare needs, are most likely to benefit from these services before, during or after care transitions $[9,11,48]$. Moreover, the literature for intermediate care highlighted the need to target older adults likely to benefit from services that offer optimal convalescence periods and transfers to the most suitable settings to prevent poor clinical and health-related outcomes $[9,14]$.

\section{Approach to care}

Consensus was established that intermediate care provides holistic and person-centred care; with the involvement of family and caregivers and support for self-management; while using relational approaches, creative solutions and simple technologies. Personalised care that resonates with what is important to meet the specific needs of older persons and family members was recommended as an ideal approach to the planning and delivery of these services in different settings. An example of this is the preventable admissions care team (PACT) intervention [49]. Operating during care transitions, this inclusive social work led programme focused on potential causes of readmission at an individual level, promoted understanding of prognosis and strategies for selfmanagement as well as communication between patients, families and healthcare professionals [49]. Intermediate care programmes combined with simple technology-based interventions such as telephone follow-up combined with shared electronic health records and care plans were reported to have positive outcomes related to reduced hospital utilisation as well as improved patient and family experiences, and self-management [16].

Delphi participants agreed that it is necessary to empower, understand and support patients and their families to enable and support self-management. Building confidence was also stressed as an important goal to enhance capacity and increase physical and social resilience. However, it was recognised that cultural disparities and differences in the organisation of healthcare systems might influence how these aspects are approached in practice. In the 
consensus meeting, some participants noted that in some countries, patients and families do not feel part of the care team, despite the emerging evidence about shared decisionmaking and treatment burden [50]. The literature emphasises that a systematic focus on patient experience could be used to improve and shape services [51]. The provision of accessible guidance and training programmes for families and informal caregivers during care transitions is important [52] given that, for example, $61 \%$ of all care activity in the United Kingdom is provided by informal caregivers and volunteers [53]. Active encouragement by healthcare professionals including clinical bedside handovers and designated family meetings or consultations at admission and discharge might be effective strategies to increase family involvement during care transitions [48, 54].

\section{Organisation of the services}

It was agreed that intermediate care is best delivered by an interdisciplinary team within an integrated health and social care system where a single point of contact might help to optimise service access, communication and coordination of care. Complementing the single point of contact with use of simple technologies was considered important to support patients, families and care givers and enhance communication. This is consistent with the literature [55] and also has potential to enable delivery of these services in economically disadvantaged environments and healthcare systems [56]. Indeed, effective intermediate care services was highlighted as a priority in the World Health Organisation's priority practices for continuity and coordination of care [57].

The participants also stressed the importance of delivering services as a network of care and support with close collaboration between healthcare, social care and voluntary and community sector partners. Key elements within the organisational model were agreed including sufficient capacity and responsiveness, appropriate expertise, clear governance arrangements, and opportunities to support the team members to work collaboratively and to improve service quality and outcomes for people and care systems. There was consensus on an urgent need to develop a workforce with confident, competent transitional care and intermediate care professionals who have the required technical and relational skills. In the literature, intermediate care delivered by interdisciplinary teams at home, in post-acute facilities, community hospitals or care homes was reported in many instances to reduce hospitalisation and improve functional outcomes [16]. Future research on resource allocation and cost effectiveness for these services is recommended, as are studies investigating the skills and competencies that are necessary for effective intermediate care.

\section{Strengths and limitations}

The study was conducted as an international e-Delphi project, which was supported by a preliminary scoping review of the literature for current definitions of intermediate care. The participants of the Delphi and virtual consensus meeting represented policy, academic and multidisciplinary subject experts (including one patient advocate) from 18 countries in total (13 participating in the Delphi) from Europe and North, Central and South America. Generation and revision of the statements were performed with maximum transparency at all stages. However, this study has some limitations. First, only themes identified during the scoping review were extracted and presented in this study; details relating to individual studies such as demographics and sampling frames were not extracted. This was due to the qualitative synthesis approach followed, which only focused on generating themes. Second, although Delphi participants supported most statements based on their personal professional experience, they could not approve the inclusion of some statements due to limited supporting scientific evidence. This is not uncommon given the inherent nature of the Delphi process, which brings together evidence from literature and personal experience, thus highlighting where gaps in the scientific evidence limit the potential to include some experiential evidence. Third, although different professions and sectors were represented, the sample did not include participants from Africa or Asia Pacific regions including Australia and New Zealand. Thus, the final consensus may not be generalisable to these regions and to low- and middle-income countries. However, studies from Asia Pacific regions were included in the scoping review that informed the process. The report of the final consensus statement was approved by the steering committee and ADVANTAGE Joint Action collaborators from 22 European member states. Therefore, the final statements include indirect representation of additional experts from Belgium, Bulgaria, Croatia, Cyprus, Germany, Hungary, Lithuania, Norway, Portugal, Romania, Slovenia, increasing the number of countries represented to 29. Fourth, the response rate for the virtual consensus meeting was low, possibly influenced by time constraints and time zone differences. In addition, an anonymised voting tool could not be used due to the continuous discussions during the meeting, time differences and technical limitations. However, all participants were encouraged to express their opinions freely and actively take part in discussions. They were also encouraged to contact the core team privately with any additional feedback on the statements. This helped to prevent overrepresentation of more senior participants in the discussions. As only one caregiver and patient advocate participated, cultural and organisational concepts were 
not fully explored from the perspectives of patients and caregivers. Fifth and finally, the cut-point for inclusion of statements was arbitrary, selected by consensus among the core group. However, there is no clear consensus on this in the literature with wide variation in cut-offs between studies $[40,43]$ and the cut-off selected here (70\%) is similar to that found in most Delphi [43].

\section{Conclusions}

This study reports the first international consensus on definitions of intermediate care, specifically examining the purpose, target population, approaches and organisation of these services. It was agreed that intermediate care represents a broad range of time-limited services that aim to ensure continuity and quality of care; promote recovery; restore independence and confidence; or prevent a decline in functional ability at the interface between hospital, home, long-term care (nursing homes), primary care and community services. Participants agreed that transitional care services are a subset of intermediate care. Such services may particularly benefit persons who have complex support needs or circumstances, are vulnerable to a decline in health status or functional ability or are at increased risk of (re)admissions to hospital or institutionalisation. Their shared approach is based on holistic and personcentred care, the involvement of family and caregivers, support for self-management, relational approaches, creative solutions and simple technologies. Intermediate care is best delivered by an interdisciplinary team within an integrated health and social care system where a single point of contact might help to optimise service access, communication and coordination of care. It was recommended that these should have sufficient capacity and responsiveness, appropriate expertise, clear governance arrangements, and opportunities to support team members to work collaboratively and to improve service quality and outcomes for people and care systems.

The consensus statement can be used by policy-makers, commissioners, academics, and professionals involved in the planning, delivery and evaluation of these services. Derived from subject experts in 18 countries in Europe and in North, Central and South America, and endorsed by policy and professional leads for older people in another 11 European member states, the consensus is highly applicable to most systems currently delivering this model of care. However, the applicability of the consensus statement should be tested in other healthcare systems, particularly in the Asia Pacific region and in low- and middle-income economies. More research is needed to understand how to translate the key approaches into practice in different care settings and within systems with varying levels of vertical and horizontal integration of health and other care services.
Funding This paper is part of Joint Action '724099/ADVANTAGE' which has received funding from the European Union's Health Programme (2014-2020).

\section{Compliance with ethical standard}

Conflict of interest On behalf of all authors, the corresponding author states that there is no conflict of interest.

Disclaimer The content of this report represents the views of the author only and is his/her sole responsibility; it cannot be considered to reflect the views of the European Commission and/or the Consumers, Health, Agriculture and Food Executive Agency or any other body of the European Union. The European Commission and the Agency do not accept any responsibility for use that may be made of the information it contains.

Statement of human and animal rights All procedures performed in this study complied with the ethical standards of the institutional research committee and with the 1964 Helsinki Declaration and its later amendments.

Informed consent All participants provided informed written (electronic) consent in advance of the study.

\section{References}

1. Mansukhani RP, Bridgeman MB, Candelario D et al (2015) Exploring transitional care: evidence-based strategies for improving provider communication and reducing readmissions. P\&T 40:690-694

2. Kripalani S, LeFevre F, Phillips CO et al (2007) Deficits in communication and information transfer between hospital-based and primary care physicians: implications for patient safety and continuity of care. JAMA 297:831-841

3. Lima M, Magalhaes AMM, Oelke ND et al (2018) Care transition strategies in Latin American countries: an integrative review. Rev Gaucha Enferm 39:e20180119

4. Coffey A, Leahy-Warren P, Savage E et al (2019) Interventions to promote early discharge and avoid inappropriate hospital (re) admission: a systematic review. Int J Environ Res Public Health 16:E2457

5. Forster AJ, Clark HD, Menard A et al (2004) Adverse events among medical patients after discharge from hospital. CMAJ 170:345-349

6. Baillie L, Gallini A, Corser R et al (2014) Care transitions for frail, older people from acute hospital wards within an integrated healthcare system in England: a qualitative case study. Int J Integr Care 14:e09

7. Allen J, Hutchinson AM, Brown R et al (2018) User experience and care for older people transitioning from hospital to home: patients' and carers' perspectives. Health Expect 21:518-527

8. Peel NM, Hubbard RE, Gray LC (2013) Impact of post-acute transition care for frail older people: a prospective study. J Frailty Aging 2:165-171

9. Loeffler K (2016) Geriatric intermediate care and transitional care for frailty-related patients: Kerstin Loeffler. Eur J Public Health 26:426-427

10. Allen J, Hutchinson AM, Brown R et al (2014) Quality care outcomes following transitional care interventions for older people from hospital to home: a systematic review. BMC Health Serv Res 15:346 
11. Lowthian $\mathbf{J}$ (2017) How do we optimise care transition of frail older people? Age Ageing 46:2-4

12. Inzitari M, Gual N, Roig T et al (2015) Geriatric screening tools to select older adults susceptible for direct transfer from the emergency department to subacute intermediate-care hospitalization. J Am Med Dir Assoc 16:837-841

13. World Health Organisation (2016) Transitions of care: technical series on safer primary care. Switzerland, Geneva

14. Coleman EA, Boult C (2003) Improving the quality of transitional care for persons with complex care needs. J Am Geriatr Soc 51:556-557

15. Naylor MD (2006) Transitional care: a critical dimension of the home healthcare quality agenda. J Healthc Qual 28:48-54

16. Sezgin D, O'Caoimh R, Liew A et al (2019) Intermediate care interventions for older adults: ADVANTAGE joint action for frailty prevention work package 7 task group report. Madrid, $p$ 1-48. https://advantageja.eu/images/WP7_Intermediate-and-trans itional-care-task-group-report.pdf. Accessed 16 Apr 2020

17. Beck AM, Kjaer S, Hansen BS et al (2013) Follow-up home visits with registered dieticians have a positive effect on the functional and nutritional status of geriatric medical patients after discharge: a randomized controlled trial. Clin Rehabil 27:483-493

18. Cunliffe AL, Gladman JR, Husbands SL et al (2004) Sooner and healthier: a randomised controlled trial and interview study of an early discharge rehabilitation service for older people. Age Ageing 33:246-252

19. Mas MA, Inzitari M, Sabate $S$ et al (2017) Hospital-at-home Integrated Care Programme for the management of disabling health crises in older patients: comparison with bed-based Intermediate Care. Age Ageing 46:925-931

20. Mas MÂ, Santaeugènia SJ, Tarazona-Santabalbina FJ et al (2018) Effectiveness of a hospital-at-home integrated care program as alternative resource for medical crises care in older adults with complex chronic conditions. J Am Med Dir Assoc 19:860-863

21. Wong FKY, Chau J, So C et al (2012) Cost-effectiveness of a healthsocial partnership transitional program for post-discharge medical patients. BMC Health Serv Res 12:1-8

22. Kind AJ, Brenny-Fitzpatrick M, Leahy-Gross K et al (2016) Harnessing protocolized adaptation in dissemination: successful implementation and sustainment of the veterans affairs coordinated-transitional care program in a non-veterans affairs hospital. J Am Geriatr Soc 64:409-416

23. Xiang X, Zuverink A, Rosenberg W et al (2019) Social work-based transitional care intervention for super utilizers of medical care: a retrospective analysis of the bridge model for super utilizers. Soc Work Health Care 58:126-141

24. Galbraith AA, Meyers DJ, Ross-Degnan D et al (2017) Long-term impact of a postdischarge community health worker intervention on health care costs in a safety-net system. Health Serv Res 52:2061-2078

25. Shah MN, Hollander MM, Jones CM et al (2018) Improving the ED-to-home transition: the community paramedic-delivered care transitions intervention-preliminary findings. J Am Geriatr Soc 66:2213-2220

26. Naylor MD, Bowles KH, McCauley KM et al (2013) High-value transitional care: translation of research into practice. J Eval Clin Pract 19:727-733

27. Manville M, Klein M, Bainbridge L (2014) Improved outcomes for elderly patients who received care on a transitional care unit. Can Fam Phys 60:e263-e271

28. Blewett LA, Johnson K, McCarthy T et al (2010) Improving geriatric transitional care through inter-professional care teams. J Eval Clin Pract 16:57-63

29. Caplan GA, Coconis J, Board N et al (2006) Does home treatment affect delirium? A randomised controlled trial of rehabilitation of elderly and care at home or usual treatment (The REACH-OUT trial). Age Ageing 35:53-60
30. Goodwin JS, Li S, Middleton A et al (2018) Differences between skilled nursing facilities in risk of subsequent long-term care placement. J Am Geriatr Soc 66:1880-1886

31. Herford JK, Heggestad T, Ersland H et al (2014) Intermediate care in nursing home after hospital admission: a randomized controlled trial with one year follow-up. BMC Res Notes 9:889

32. Melis RJF, Rikkert MGMO, Parker SG et al (2004) What is intermediate care? BMJ 329:360-361

33. Martin GP, Peet SM, Hewitt GJ et al (2004) Diversity in intermediate care. Health Soc Care Commun 12:150-154

34. Colquhoun HL, Levac D, O'Brien KK et al (2014) Scoping reviews: time for clarity in definition, methods, and reporting. J Clin Epidemiol 67:1291-1294

35. Levac D, Colquhoun H, O'Brien KK (2010) Scoping studies: advancing the methodology. Implement Sci 5:69

36. Tricco AC, Lillie E, Zarin W et al (2018) PRISMA extension for scoping reviews (PRISMA-ScR): checklist and explanation. Ann Intern Med 169:467-473

37. Lockwood C, Munn Z, Porritt K (2015) Qualitative research synthesis: methodological guidance for systematic reviewers utilizing meta-aggregation. Int J Evid Based Healthc 13:179-187

38. Hanafin S (2004) Review of literature on the Delphi Technique. In: Affairs DoCaY (ed) Ireland: National Children's Office, p. 1-54

39. Linstone HA, Turoff M (2002) The Delphi method techniques and applications. Newark. https://web.njit.edu/ turoff/pubs/delphibook /delphibook.pdf. Accessed 16 Apr 2020

40. Giannarou L, Zervas E (2014) Using Delphi technique to build consensus in practice. Int J Bus Sci Appl Manag 9:65-82

41. Hendry A, Vanhecke E, Carriazo AM et al (2019) Integrated care models for managing and preventing frailty: a systematic review for the European Joint Action on Frailty Prevention (ADVANTAGE JA). Transl Med UniSa 19:5-10

42. Hsu CC, Sandford BA (2007) The Delphi technique: making sense of consensus. Pract Assess Res Eval 12:1-8

43. Diamond IR, Grant RC, Feldman BM et al (2014) Defining consensus: a systematic review recommends methodologic criteria for reporting of Delphi studies. J Clin Epidemiol 67:401-409

44. Steiner A (1997) Intermediate care: a conceptual framework and review of the literature. King's Fund, London

45. Griffiths PD, Edwards MH, Forbes A et al (2007) Effectiveness of intermediate care in nursing-led in-patient units. Cochrane Database Syst Rev 2:CD002214

46. Kansagara D, Chiovaro JC, Kagen D et al (2016) So many options, where do we start? An overview of the care transitions literature. J Hosp Med 11:221-230

47. Shahsavari H, Zarei M, Aliheydari MJ (2019) Transitional care: concept analysis using Rodgers' evolutionary approach. Int J Nurs Stud 99:103387

48. Jeffs L, Saragosa M, Law MP et al (2017) The role of caregivers in interfacility care transitions: a qualitative study. Patient Prefer Adher 11:1443-1450

49. Weerahandi $\mathrm{H}$ et al (2015) Effects of a psychosocial transitional care model on hospitalizations and cost of care for high utilizers. Soc Work Health Care 54:485-498

50. Rosbach M, Andersen JS (2017) Patient-experienced burden of treatment in patients with multimorbidity - a systematic review of qualitative data. PLoS ONE 12:e179916

51. Hasardzhiev S, Mendao L, Nolte W et al (2016) Managing multimorbidity: how can the patient experience be improved? J Comorb 6:28-32

52. Gibson MJ, Kelly KA, Kaplan AK (2012) Family caregiving and transitional care: a critical review. In: U.S. Department of Health and Human Services AfCL, Administration on Aging (ed) The National Center on Caregiving, Family Caregiver Alliance, USA, p. 1-54

53. Willis G, Edwards M, Lyscom T, et al (2015) Future demand for skills: initial results. In: Health Do (ed) The Centre for Workforce Intelligence (CfWI), United Kingdom, p. 13 
54. Manias E, Bucknall T, Hughes C et al (2019) Family involvement in managing medications of older patients across transitions of care: a systematic review. BMC Geriatr 19:95

55. Kansagara D, Chiovaro JC, Kagen D, et al (2015) Transitions of care from hospital to home: an overview of systematic reviews and recommendations for improving transitional care in the veterans health administration. In: Service DoVAHSRD (ed) Quality Enhancement Research Initiative, Washington, p. 1-62

56. Feuerwerker LCM, Merhy EE (2008) A contribuição da atenção domiciliar para a configuração de redes substitutivas de saúde: desinstitucionalização e transformação de práticas. Rev Panam Salud Publica 24:180-188
57. World Health Organisation (2018) Continuity and coordination of care: A practice brief to support implementation of the WHO Framework on integrated people-centred health services. World Health Organisation, Geneva

Publisher's Note Springer Nature remains neutral with regard to jurisdictional claims in published maps and institutional affiliations.

\section{Affiliations}

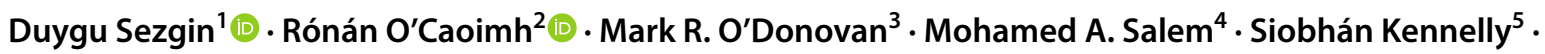
Luz Lopez Samaniego ${ }^{6}$. Cristina Arnal Carda ${ }^{7}$. Rafael Rodriguez-Acuña ${ }^{6} \cdot$ Marco Inzitari $^{8}$. Teija Hammar ${ }^{9}$. Claire Holditch $^{10}$. Janet Prvu Bettger ${ }^{11}$. Martin Vernon ${ }^{12,13}$. Áine Carroll ${ }^{14,15}$. Felix Gradinger ${ }^{16,17}$.

Gaston Perman $^{18} \cdot$ Martin Wilson $^{19} \cdot$ Antoine Vella $^{20}$. Antonio Cherubini ${ }^{21} \cdot$ Helen Tucker $^{22} \cdot$ Maria Pia Fantini $^{23}$. Graziano Onder $^{24} \cdot$ Regina Roller-Wirnsberger ${ }^{25} \cdot$ Luis Miguel Gutiérrez-Robledo ${ }^{26} \cdot$ Matteo Cesari $^{27}$. Paula Bertoluci ${ }^{28}$. Magdalena Kieliszek ${ }^{29}$. Wilma van der Vlegel-Brouwer ${ }^{30}$. Michelle Nelson ${ }^{31,32}$. Leocadio Rodríguez Mañas ${ }^{33}$. Eleftheria Antoniadou ${ }^{34}$. François Barriere ${ }^{35}$. Sebastian Lindblom ${ }^{36}$. Grace Park ${ }^{37}$. Isidoro Pérez ${ }^{38}$. Dolores Alguacil ${ }^{39}$. Douglas Lowdon ${ }^{40}$. Maria Eugenia Alkiza ${ }^{41}$. Cristina Alonso Bouzon ${ }^{42}$. John Young ${ }^{43}$. Ana Carriazo ${ }^{44}$. Aaron Liew ${ }^{45,46}$. Anne Hendry ${ }^{47,48}$ on behalf of European Union Advantage Joint Action Work Package 7 partners in collaboration with the International Foundation for Integrated Care Special Interest Group on Intermediate Care

Rónán O’Caoimh

rocaoimh@hotmail.com

1 College of Medicine, Nursing and Health Sciences, National University of Ireland Galway, Galway City, Ireland

2 Department of Geriatric Medicine, Mercy University Hospital Cork, Cork City, Ireland

3 Clinical Sciences Institute, National University of Ireland Galway, Galway City, Ireland

4 St Vincent De Paule LTC Facility, Marsa, Malta

5 Older Person's Programme, Health Service Executive, Dublin, Ireland

6 Andalusian Public Foundation of Progress and Health (FPS), Seville, Spain

7 Hospital a Domicili Integral Geriatria (HaDo), Barcelona, Spain

8 Parc Sanitari Pere Virgili, Vall D’Hebrón Institute of Research (VHIR), and Universitat Autònoma de Barcelona, Barcelona, Spain

9 Finnish Institute for Health and Welfare (THL), Helsinki, Finland

10 NHS Benchmarking Network, Manchester, England

11 Duke University, Durham, USA

12 NHS England, London, England

13 Manchester University NHS Foundation Trust, London, England

14 University College Dublin, Dublin, Ireland
15 National Rehabilitation Hospital, Dún Laoghaire, County Dublin, Ireland

16 University of Plymouth, Plymouth, England

17 Torbay and South Devon NHS Foundation Trust, Torbay, England

18 Hospital Italiano de Buenos Aires, Buenos Aires, Argentina

19 Raigmore Hospital Inverness NHS Highland, Inverness, Scotland

20 Department of Geriatric Medicine, University of Malta, Msida, Malta

21 Geriatria, Accettazione geriatrica e Centro di Ricerca Per L'invecchiamento, IRCCS INRCA, Ancona, Italy

22 Community Hospitals Association (UK), University of Winchester, Winchester, England

23 Post-Graduate Medical School in Hygiene and Preventive Medicine, Department of Biomedical and Neuromotor Sciences, Alma Mater Studiorum-University of Bologna, Bologna, Italy

24 Department of Cardiovascular, Endocrine-Metabolic Diseases and Aging, Istituto Superiore di Sanità, Rome, Italy

25 Department of Internal Medicine, Medical University of Graz, Graz, Austria

26 National Institute of Geriatric Medicine at the National Institutes of Health, Mexico City, Mexico

27 Fondazione IRCCS Ca' Granda Ospedale Maggiore Policlinico, Università Degli Studi di Milano, Milan, Italy

28 Alves Pereira, Public Health School, University of São Paulo (FSP/USP), São Paulo, Brazil 
29 Center for Innovation and Technology Transfer, Medical University of Lodz, Lodz, Poland

30 HU University of Applied Sciences, Utrecht, The Netherlands

31 Lunenfeld-Tanenbaum Research Institute, Toronto, Canada

32 Institute of Health Policy, Management and Evaluation, University of Toronto, Toronto, Canada

33 Jefe de Servicio de Geriatría Hospital Universitario de Getafe, Getafe, Spain

34 Rehabilitation Clinic Patras University Hospital, Patras, Greece

35 Pilote MAIA 13 Projets Parcours Pays Salonais, Pays Salonais, France

36 Department of Neurobiology, Care Sciences and Society, Karolinska Institutet, Huddinge, Sweden

37 Fraser Health Authority, British Columbia, Canada

38

\author{
Regional Ministry of Health and Families of Andalusia \\ (CSFJA), Seville, Spain
}

39 Andalusian Health Service (Servicio Andaluz de Salud, SAS), Seville, Spain

40 NHS Scotland, Edinburgh, Scotland

41 Bidasoa Integrated Healthcare Organisation, Osakidetza, Basque Country, Spain

42 The Spanish Society of Geriatrics Medicine (SEMEG), Madrid, Spain

43 University of Leeds, Leeds, England

44 Regional Ministry of Health and Families of Andalusia (CSFJA), Seville, Spain

45 Clinical Sciences Institute, National University of Ireland Galway, Galway, Ireland

46 Department of Endocrinology, Portiuncula University Hospital, Ballinasloe, Co Galway, Ireland

47 NHS Lanarkshire, Glasgow, Scotland

48 University of the West of Scotland, Glasgow, Scotland 